\title{
HIV DRUG RESISTANCE TENDENCIES IN LATVIA
}

\author{
Tatjana Kolupajeva ${ }^{1}$, Pauls Aldins ${ }^{1}$, Ludmila Guseva ${ }^{1}$, Diana Dusacka ${ }^{1}$, Valentina Sondore ${ }^{1}$, Ludmila Viksna ${ }^{2}$, \\ Baiba Rozentale ${ }^{1}$ \\ ${ }^{1}$ State Agency Infectology Center of Latvia, Riga, Latvia \\ ${ }^{2}$ Riga Stradins University, Riga, Latvia
}

\section{SUMMARY}

The treatment of HIV infection in Latvia by using highly active antiretroviral therapy (HAART) was started in 1996.

The prevalence and tendencies of HIV drug resistance among treated and treatment-naive patients in Latvia in the years 2006-2007 were evaluated in this study. Data of HIV genotyping, performed in 132 HIV-1 infected during years 2006-2007 by TRUGENE HIV-1 genotyping assay (BayerHealthCare-diagnostics) are included in the study.

Analysis of data showed that in the group of treatment-naive individuals majority carried wild type virus. Prevalence of resistance-associated mutations (RAMs) in the treatment-naive group according to IAS list was $28 \%$. In most cases it was NRTI mutation A62V that is associated with multinucleoside resistance caused by Q151M, its effect in the absence of Q151M is not known. By many authors A62V is supposed to be a result of polymorphism in RT gene and is excluded from the list of resistance mutations. High prevalence of A62V is typical for HIV-1 subtype A. As majority of treatment-naive cases (89\%) in this study were with HIV-1 subtypes A or AE, we excluded A62V mutation and estimated RAMs prevalence in group of treatment-naive HIV-infected individuals as 7\%. Minor PI mutations were not included in analyses. In Europe published rates generally very between $5 \%$ and $15 \%$.

In the group of treatment-experienced HIV infected people 25/75 were with HIV-1 subtype B, the rest part - with non-B subtypes: A/AE (35/75), CRF - 01AE (7/75), B/AE (4/75) and others. In treatment-experienced patients RAMs prevalence was estimated as 58.6\%. Most frequently RAMs were found for nucleoside reverse transcriptase inhibitors (NRTI) (49.3\%) followed by non-nucleoside reverse transcriptase inhibitors (NNRTI) (22.6\%) and protease inhibitors (PI) (16\%).

In the group of NRTI mutations M184V (26/75; 34.6\%), A62V (12/75; 16.0\%) and T215Y (8/75; 10.6\%), in NNRTI mutations K103N (10/75; 13.3\%), G190S (6/75; 8.0\%), in PI group mutations L90M (6/75; 8.0\%) and M461/L (6/75; 8.0\%) occurred most frequently.

The following drug susceptibility was predicted according to the Trugen expert interpretations: in 33/75 (44\%) patients no evidence of resistance, in 21/75 (28\%) patients resistance to 1 drug class (NRTI - 16/75 NNRTI - 4/75, PI - 1/75), in 17 patients (22.6\%) resistance to 2 drug classes (NRTI+NNRTI - 9/75, NRTI+PI - 7/75, NNRTI+PI - 1/75) and in 3/75 (4\%) patients resistance to all 3 classes of drugs (NRTI+NNRTI+PI).

We conclude, that prevalence of RAMs in treatment-naive HIV infected persons in Latvia is comparable with prevalence in Europe. The origin of predominated mutation A62V associated with NRTI at present is not clear. In more than half of treated HIV infected patients HIV resistance to at least one HAART class was predicted.

Key words: HIV infection, genotyping, drug resistance, mutations, HAART

Address for correspondence: V. Sondore, 3, Linezera street, Riga, LV-1006. E-mail: valentina.sondore@lic.gov.lv

\section{INTRODUCTION}

HIV infection has been recognized in Latvia since 1987, when the first HIV-1 infected person was registered. During the following ten years HIV transmission occurred mainly via sexual contacts. However, due to the epidemic among injecting drug users (IDUs) started in 1997, the number of newly detected HIV-infected patients increased considerably and reached the maximum in 2001, but now is slowly declining. By November 2007, 3,921 HIV infection cases had been reported in Latvia: 476 patients have developed AIDS and 227 patients have died. Treatment by monotherapy was started in 1990, highly active antiretroviral therapy (HAART) - in 1996.

The number of HAART, their recommended combinations, timing and dosage change almost continuously. The current com- bination therapies are not effective for many people. Analysis of literature data shows that in case of HIV, HAART can increase HIV mutation rates (1). Thus, drug resistance arised as a longterm dilemma.

Interrupting of the therapy course or failure to take anti-HIV drugs on schedule and in the prescribed dosage can encourage the development of drug resistant HIV strains. Every missed dose is an accelerating factor for drug resistance emerging.

Many analyses of the evaluation of HIV population within infected hosts have shown that, starting from a homogenous virus population early after infection, the frequency of mutants with mutations at certain positions increases over time, even without any therapeutical interference.

HIV drug therapy often fails because of appearance of multidrug-resistant virus. Resistant HIV may preexist at low concentra- 
tions in drug-naive patients (infected with HIV resistant mutant or developed HIV mutant during the natural course of host-pathogen interaction?) and is rapidly selected in the presence of drugs. The other scenario - resistant virus is absent at the start of therapy, but is generated by residual viral replication during therapy (2). Both acquired and transmitted HIV-1 drug resistance are public health concerns, but transmitted resistance has the potential to more rapidly reverse the effectiveness of first-line antiretroviral therapy at the population level. Surveillance of both transmitted and acquired resistance can supply information to support public health bodies in designing preventive action to minimize the development and transmission of drug-resistant viruses.

The objective of this study was to evaluate the prevalence and tendencies of HIV drug resistance among treated and treatment-naive patients in Latvia in the years 2006-2007.

\section{MATERIALS AND METHODS}

Laboratory monitoring of treatment efficacy from 1994 was available only by using flow cytometry for CD4 and CD8 cells counting and HIV viral load detection by quantitative PCR. Since 2004, HIV drug resistance testing by commercial kits VERSANT HIV-1 resistance (LiPA) was available. The method is based on the molecular hybridization principle and was limited to the resistance-associated mutations (RAMs) known at the moment. That is why method based on the sequencing - TRUGENE HIV-1 genotyping assay (BayerHealthCare-diagnostics) was introduced since late 2005.

According to national "HIV infection treatment guidelines" (August, 2006), HIV resistance testing is performed in patients with treatment failure as well as before initiating of therapy. Besides the prognosis for the correction of therapy for the patients, summary of these data allows us to evaluate the prevalence of resistance-associated mutations and more common patterns of mutant combinations in Latvia.

Data of HIV genotyping, performed in 2006-2007 by TRUGENE HIV-1 genotyping assay, are included in the study. Quality of testing was confirmed by participation in the QCMD programme. Drug resistance testing was performed in 132 HIV-1 infected individuals: 57 treatment-naive individuals were tested before starting therapy (34 males and 23 females), and 75 individuals with treatment failure (51 males and 24 females, treatment course from 1 till 15 years). HIV transmission ways in treatment-naive group were the following: intravenous drug use (30/57), through heterosexual (22/57) and homosexual (4/57) contacts, vertical transmission (1/57); in treatment-experienced group: intravenous drug use (26/75), through heterosexual (18/75) and homosexual (22/75) contacts, vertical transmission (7/75) and unknown (2/75).

For the estimation of prevalence RAMs were included as defined by the International AIDS Society (IAS) guidelines, updated in 2007 (3). Subtyping was performed by using Stanford database.

\section{RESULTS}

In the group of treatment-naive individuals majority (42/57; $73.6 \%$ ) carried wild type virus, in 16 out of 57 sequences at least
1 RAM was found. RAMs prevalence according to IAS list in the treatment-naive group was $28 \%$. In most cases (13/16) it was NRTI mutation A62V that is associated with multinucleoside resistance caused by Q151M, its effect in the absence of Q151M is not known. Minor PI mutations were not included in analyses.

Almost all treatment-naive cases (51/57) were with HIV-1 nonB subtypes A or AE, subtype B was found only in 6/57 cases.

In the group of treatment-experienced HIV infected people 25/75 were with HIV-1 B subtype, the rest part - with non-B subtypes: A/AE (35/75), CRF - 01AE (7/75), B/AE (4/75) and others.

In treatment-experienced patients RAMs prevalence was estimated as $58.6 \%(44 / 75)$. Most frequently RAMs were found for NRTI (37/75; 49.3\%) followed by NNRTI (17/75; 22.6\%) and PI (12/75; 16\%).

In the group of NRTI mutations M184V (26/75; 34.6\%), A62V (12/75; 16.0\%) and T215Y (8/75; 10.6\%), in NNRTI mutations K103N (10/75; 13.3\%), G190S (6/75; 8.0\%), in PI group mutations L90M (6/75; 8.0\%), M461/L (6/75; 8.0\%) occurred most frequently (Table 1).

The following drug susceptibility was predicted according to the Trugene expert interpretations: in 33/75 (44\%) patients no evidence of resistance, in 21/75 (28\%) patients resistance to 1 drug class (NRTI - 16/75 NNRTI - 4/75, PI - 1/75), in 17 patients (22.6\%) resistance to 2 drug classes (NRTI+NNRTI - 9/75, NRTI+PI - 7/75, NNRTI+PI - 1/75) and in 3/75 (4\%) patients resistance to all 3 classes of drugs (NRTI+NNRTI+PI) (see Fig. 1).

Table 1. More frequent resistance-associated mutations (RAMs) in treatment-experienced patients

\begin{tabular}{|l|c|c|}
\hline \multirow{2}{*}{ RAMs group } & \multicolumn{2}{|c|}{ RAMs prevalence } \\
\cline { 2 - 3 } & number & $\%$ \\
\hline NRTI & $26 / 75$ & 34.6 \\
\hline M184V & $12 / 75$ & 16.0 \\
\hline A62V & $8 / 75$ & 10.6 \\
\hline T215Y & $10 / 75$ & 13.3 \\
\hline NNRTI & $6 / 75$ & 8.0 \\
\hline K103N & \multicolumn{2}{|}{} \\
\hline G190S & $6 / 75$ & 8.0 \\
\hline PI & $6 / 75$ & 8.0 \\
\hline L90M & \multicolumn{2}{|l}{} \\
\hline M461 & \multicolumn{2}{|l}{} \\
\hline
\end{tabular}

\section{DISCUSSION}

In the group of treatment-naive HIV infected individuals prevalence of RAMs according to IAS list 2007 was found rather high - 28\%, most frequently NRTI group mutation A62V was found. But in spite of fact that IAS list is used by many surveillance studies in Europe, we should take in consideration the opinion that by many authors $\mathrm{A} 62 \mathrm{~V}$ is believed to be a result of polymorphism in RT gene and is excluded from the list of resistance mutations (4). High prevalence of HIV-1 subtype A with A62V polymorphism 


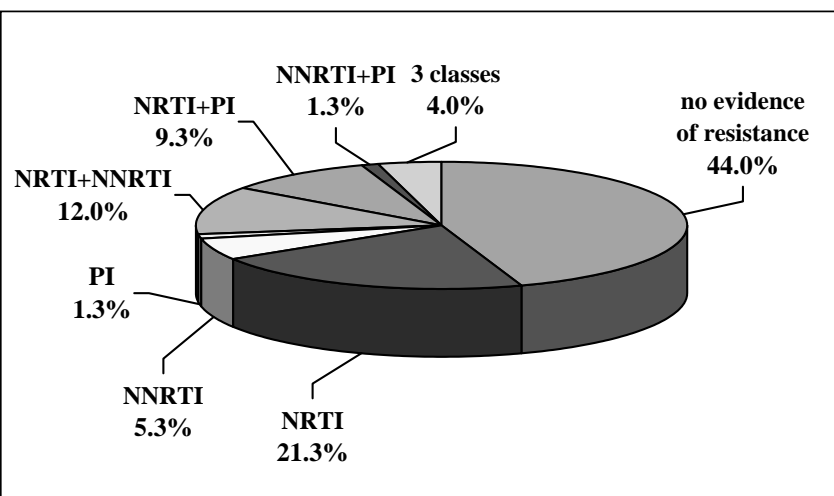

Fig. 1. Resistance to antiretroviral drugs among treatmentexperienced patients. NRTi-nucleoside reverse transcriptase inhibitors, NNRTi-non-nucleoside reverse transcriptase inhibitors, PI-protease inhibitors.

in RT gene was found in Russia. According to the Stanford HIV Drug resistance database these mutations prevalence in HIV-1 subtype A is $17 \%$, in subtype $\mathrm{AE}-6 \%$. As majority of studied treatment-naive cases (89\%) were with HIV-1 subtypes A or AE, we excluded $\mathrm{A} 62 \mathrm{~V}$ mutation and estimated RAMs prevalence in group of treatment-naive HIV-infected individuals as only $7 \%$, that is comparable with available data - rates generally between $5 \%$ and $15 \%$ (2) - in the Europe. In 56\% (42/75) of treated individuals resistance to at least one drug class was predicted.

No difference in RAMs prevalence was observed among males and females, neither treatment naive nor treatment experienced.

Most of mutations weaken the virus, and some have no impact at all. But a few patterns of mutation have the effect of making the virus less susceptible to certain drugs. Accumulation of mutations, including PI minor mutations (that are not analyzed in this study) may result in a lower genetic barrier to resistance and reduced response to the drugs that were not used for the treatment of HIV-infected person. Thus, the prognosis of possible resistance to tipranavir + ritonavir combination in patients who never received these drugs was observed.

Genotypic testing used in this study identified mutations in HIV samples from an individual. At the same time, doctors really at present are still learning how best and exactly to interpret the results of these genotypic tests to improve the treatment regimens for every individual HIV-infected patient. There is still much controversy about genotypic and phenotypic testing accuracy as diagnostic tools and their usefulness to predict a treatment success. Moreover, the in silico simulations and mathematical approaches to investigate many of the various aspects of HIV infection are used, but such approach is still at an early stage (5). A stochastic model for the analysis of HIV evolution was developed that in some details reflects both the biology of HIV replication and the infection process within a host. The model provides a versatile platform for predicting the response of HIV towards therapeutic interventions and demonstrating the variations in the virus mutant dynamics - from de novo arising to preexisting at the time of treatment.

Consistently with the observations of Chen et al. (6) our data support the general thesis that the NRTIs currently used in antiviral drug therapy increase HIV mutation frequencies. The current management of HIV-1 infection involves combinations of NRTIs, NNRTIs and PIs that are changed over time when drug resistance occurs. The data discussed here reveals that increased HIV-1 mutation rates can be associated with the evolution of drug resistance (7).

\section{CONCLUSIONS}

1. Prevalence of RAMs in treatment-naive HIV infected persons in Latvia is comparable with prevalence in Europe. The origin of predominated mutation A62V associated with NRTI at present is not clear.

2. In more than half of treated HIV-infected patients HIV resistance to at least one HAART class was predicted.

\section{Acknowledgements}

This study was supported by finances of Project No. 10 of National Program No. 8 and Project No. 06.0035.05 of Latvian Council of Science.

\section{REFERENCES}

1. Mascolini M, Schapiro J. Clinical implications of resistance to new antiretrovirals: key data from the fourth European HIV drug resistance workshop. Rev Antivir Ther. 2006;2:4-14.

2. Sagir A, Oette M, Kaiser R, Däumer M, Fätkenheuer G, Rockstroh JK, et al. Trends of prevalence of primary HIV drug resistance in Germany. J Antimicrob Chemother. 2007 Oct;60(4):843-8.

3. Johnson VA, Brun-Vézinet F, Clotet B, Günthard HF, Kuritzkes DR, Pillay D, et al. Update of the drug resistance mutations in HIV-1: 2007. Top HIV Med. 2007 Aug-Sep;15(4):119-25.

4. Shafer RW, Rhee SY, Pillay D, Miller V, Sandstrom P, Schapiro JM, et al. HIV-1 protease and reverse transcriptase mutations for drug resistance surveillance. AIDS. 2007 Jan 11;21(2):215-23.

5. Bocharov G, Ford NJ, Edwards J, Breinig T, Wain-Hobson S, Meyerhans A. A genetic-algorithm approach to simulating human immunodeficiency virus evolution reveals the strong impact of multiply infected cells and recombination. J Gen Virol. 2005 Nov;86(Pt 11):3109-18.

6. Chen R, Yokoyama M, Sato H, Reilly C, Mansky LM. Human immunodeficiency virus mutagenesis during antiviral therapy: impact of drug-resistant reverse transcriptase and nucleoside and nonnucleoside reverse transcriptase inhibitors on human immunodeficiency virus type 1 mutation frequencies. J Virol. 2005 Sep;79(18):12045-57.

7. Jewell NA, Chen R, Raices R, Mansky LM. Nucleoside reverse transcriptase inhibitors and HIV mutagenesis. J Antimicrob Chemother. 2003 Oct;52(4):547-50.

Received January 9, 2008 Accepted in revised form June 20, 2008 